\title{
Restricted Diffusion
}

National Cancer Institute

\section{Source}

National Cancer Institute. Restricted Diffusion. NCI Thesaurus. Code C82346.

Transport of substances across a biological membrane where the flux rate of the diffusing material is controlled by the permeability of the membrane, which in turn is dictated by the size of the pores. 